\title{
Correction: Ethnic differences in maternal diet in pregnancy and infant eczema
}

\section{The PLOS ONE Staff}

\section{Notice of Republication}

This article was republished on May 22, 2020, to correct errors in the byline that were introduced during the typesetting process. The publisher apologizes for the errors. Please download this article again to view the correct version. The originally published, uncorrected article and the republished, corrected articles are provided here for reference.

\section{Supporting information}

S1 File. Originally published, uncorrected article. (PDF)

S2 File. Republished, corrected article.

\section{Reference}

1. Zulyniak MA, de Souza RJ, Shaikh M, Ramasundarahettige C, Tam K, Williams N, et al. (2020) Ethnic differences in maternal diet in pregnancy and infant eczema. PLoS ONE 15(5): e0232170. https://doi. org/10.1371/journal.pone.0232170 PMID: 32407330

\section{Gopenaccess}

Citation: The PLOS ONE Staff (2021) Correction: Ethnic differences in maternal diet in pregnancy and infant eczema. PLoS ONE 16(1): e0246481. https://doi.org/10.1371/journal.pone.0246481

Published: January 28, 2021

Copyright: ๑ 2021 The PLOS ONE Staff. This is an open access article distributed under the terms of the Creative Commons Attribution License, which permits unrestricted use, distribution, and reproduction in any medium, provided the original author and source are credited. 